\title{
Neutron and nuclear inelastic scattering study of the Einstein oscillators in Ba-, Sr-, and Eu-filled germanium clathrates
}

\author{
Raphaël P. Hermann,,${ }^{1, *}$ Werner Schweika, ${ }^{2}$ Olaf Leupold,${ }^{3, \dagger}$ Rudolf Rüffer, ${ }^{3}$ George S. Nolas, ${ }^{4}$ Fernande Grandjean, ${ }^{1}$ and \\ Gary J. Long ${ }^{5}$ \\ ${ }^{1}$ Department of Physics, B5, University of Liège, B-4000 Sart-Tilman, Belgium \\ ${ }^{2}$ Institut für Festkörperforschung, Forschungzentrum Jülich GmbH, D-52425 Jülich, Germany \\ ${ }^{3}$ European Synchrotron Radiation Facility, BP 220, F-38043 Grenoble, France \\ ${ }^{4}$ Department of Physics, University of South Florida, Tampa, Florida 33620, USA \\ ${ }^{5}$ Department of Chemistry, University of Missouri-Rolla, Rolla, Missouri 65409-0010, USA
}

(Received 30 March 2005; revised manuscript received 9 August 2005; published 3 November 2005)

\begin{abstract}
Inelastic neutron scattering measurements on $\mathrm{Ba}_{8} \mathrm{Ga}_{16} \mathrm{Ge}_{30}$ and $\mathrm{Sr}_{8} \mathrm{Ga}_{16} \mathrm{Ge}_{30}$ indicate the presence of soft local modes with Einstein oscillator energies of $4.9 \pm 0.1$ and $4.4 \pm 0.1 \mathrm{meV}$, modes which are associated with the lowest "rattling" mode of the $\mathrm{Ba}(\mathrm{B})$ and $\mathrm{Sr}(\mathrm{B})$ guests, respectively. Nuclear inelastic scattering measurements on $\mathrm{Eu}_{8} \mathrm{Ga}_{16} \mathrm{Ge}_{30}$ yield Einstein oscillator energies of between $3.0 \pm 0.3$ and $7.5 \pm 1.2 \mathrm{meV}$ for the different modes of the Eu guests. Further, the resonant character of the measurements on $\mathrm{Eu}_{8} \mathrm{Ga}_{16} \mathrm{Ge}_{30}$ reveals, without question, that neither of the Eu guests exhibit any vibrational modes above $9 \mathrm{meV}$.
\end{abstract}

DOI: 10.1103/PhysRevB.72.174301

PACS number(s): 63.20.Pw, 78.70.Nx, 78.70.Ck

\section{INTRODUCTION}

The dynamics of guests trapped in crystalline cages plays a crucial role in enhancing the properties of various thermoelectric materials. ${ }^{1-6}$ The weakly bound guests "rattle" in their cage and this "rattling" significantly impedes thermal transport in the crystal without impeding electrical transport. A material containing such caged guests thus behaves both as a phonon glass and an electron crystal. The "rattling" guests are probably the simplest illustration of the importance of Einstein's first paper on the quantum theory of solids, a paper in which the solid is modeled as a collection of independently vibrating harmonic oscillators. ${ }^{7}$ In filled skutterudites, a class of such caged materials, the "rattling" guests are known to behave as Einstein oscillators that are believed to effectively scatter the heat transporting phonons. ${ }^{5,6}$

The filled clathrates studied herein, $\mathrm{Ba}_{8} \mathrm{Ga}_{16} \mathrm{Ge}_{30}$, $\mathrm{Sr}_{8} \mathrm{Ga}_{16} \mathrm{Ge}_{30}$, and $\mathrm{Eu}_{8} \mathrm{Ga}_{16} \mathrm{Ge}_{30}$, belong to a different family of thermoelectric materials, a family in which the dynamics of the guests trapped in the gallium and germanium cages is responsible for the material's low thermal conductivity. ${ }^{1}$ The "rattling" behavior of the guests has also been used to explain the temperature dependence of the heat capacity ${ }^{8}$ and the Raman scattering ${ }^{9}$ observed in these filled galliumgermanium type-I clathrates. In these filled $R_{8} \mathrm{Ga}_{16} \mathrm{Ge}_{30}$ clathrates the $R$ guests occupy two cages,${ }^{10}$ the first guest, $R(\mathrm{~A})$, is located at the center of the smaller, dodecahedral cage, whereas the second guest, $R(\mathrm{~B})$, is located at the center of the larger, tetrakaidecahedral cage; the ratio of the site occupancies, $R(\mathrm{~A}): R(\mathrm{~B})$, is $1: 3$. Both the $R(\mathrm{~A})$ and $R(\mathrm{~B})$ guests are expected to behave as Einstein oscillators. ${ }^{8,9}$

Inelastic neutron scattering has proven to be a valuable technique ${ }^{5,6}$ for determining the phonon density of states of a material, for obtaining evidence for a "rattling" mode, and for measuring the Einstein oscillator energy of this mode. Herein, the method previously used by Hermann et al. ${ }^{6}$ is used to obtain the Einstein oscillator energies of the $R(\mathrm{~B})$ guests in $\mathrm{Ba}_{8} \mathrm{Ga}_{16} \mathrm{Ge}_{30}$ and $\mathrm{Sr}_{8} \mathrm{Ga}_{16} \mathrm{Ge}_{30}$. A similar study of the europium guests in $\mathrm{Eu}_{8} \mathrm{Ga}_{16} \mathrm{Ge}_{30}$ is not possible because europium has a very large neutron absorption cross section.

Fortunately, europium-151 is a Mössbauer active nuclide, and the europium partial phonon density of states can be obtained by nuclear inelastic scattering, a recently developed technique that permits the measurement of the element specific partial phonon density of states, but only for solids containing Mössbauer active nuclides. ${ }^{11-14}$ This technique utilizes the high brilliance of synchrotron radiation, combined with high resolution monochromators, to obtain an element specific vibrational density of states. In contrast to Mössbauer absorption spectroscopy, in which the recoil free absorption cross section is measured, with nuclear inelastic scattering the phonon assisted resonant nuclear absorption cross section is measured by observing the delayed fluorescence of the excited Mössbauer active nuclide. The phonon density of states of the nuclide undergoing the nuclear resonant fluorescence is obtained by an energy scan above and below the nuclear resonance. Unlike inelastic neutron scattering, nuclear inelastic scattering has the added advantage that the nuclear fluorescence yields an ideal averaging over the entire Brillouin zone. ${ }^{13,15}$ Herein, this method is used to obtain the Einstein oscillator energies of the Eu guests in $\mathrm{Eu}_{8} \mathrm{Ga}_{16} \mathrm{Ge}_{30}$.

\section{EXPERIMENT}

The samples of $\mathrm{Ba}_{8} \mathrm{Ga}_{16} \mathrm{Ge}_{30}, \quad \mathrm{Sr}_{8} \mathrm{Ga}_{16} \mathrm{Ge}_{30}, \quad$ and $\mathrm{Eu}_{8} \mathrm{Ga}_{16} \mathrm{Ge}_{30}$ have been prepared and characterized as reported earlier. ${ }^{2,10}$ It should be noted that the $\mathrm{Eu}_{8} \mathrm{Ga}_{16} \mathrm{Ge}_{30}$ clathrate studied herein has the type-I structure ${ }^{8}$ and not the type-VIII structure. ${ }^{4}$

Inelastic neutron scattering measurements have been carried out at $295 \mathrm{~K}$ on 2.81 and $1.34 \mathrm{~g}$ of polycrystalline $\mathrm{Ba}_{8} \mathrm{Ga}_{16} \mathrm{Ge}_{30}$ and $\mathrm{Sr}_{8} \mathrm{Ga}_{16} \mathrm{Ge}_{30}$, respectively, on the DNS cold neutron time of flight instrument at the DIDO research reac- 


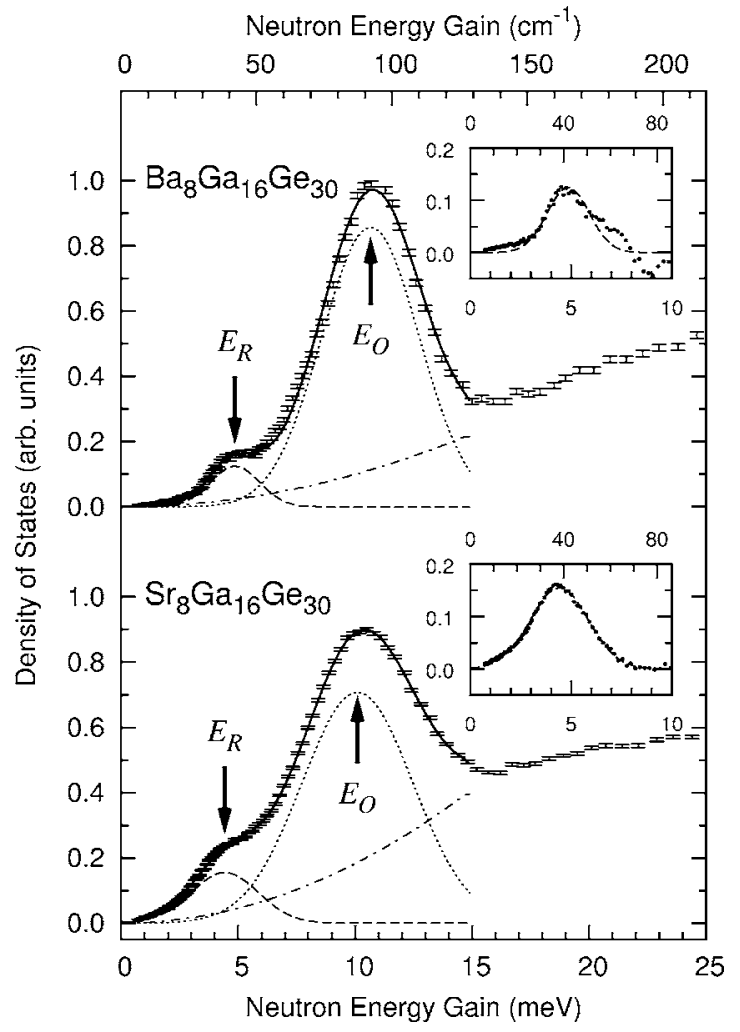

FIG. 1. The phonon density of states obtained from inelastic neutron scattering by $\mathrm{Ba}_{8} \mathrm{Ga}_{16} \mathrm{Ge}_{30}$ and $\mathrm{Sr}_{8} \mathrm{Ga}_{16} \mathrm{Ge}_{30}$. The solid line is a fit with Eq. (1), the dashed line is the contribution of the $\mathrm{Ba}(\mathrm{B})$ and $\operatorname{Sr}(\mathrm{B})$ "rattling" modes, the dotted-dashed line is the parabolic Debye contribution, and the dotted line is a Gaussian fit to the optical phonon density of states below $15 \mathrm{meV}$. Because both the neutron scattering lengths of barium and strontium differ and the framework dynamics may be different in the two compounds, the measured DOS have been arbitrarily scaled. Insets: The Gaussian peaks at $E_{R}$ associated with the $\mathrm{Ba}(\mathrm{B})$ and $\mathrm{Sr}(\mathrm{B})$ "rattling" modes. Deviations from the simplified model, see inset for $\mathrm{Ba}_{8} \mathrm{Ga}_{16} \mathrm{Ge}_{30}$, might be related to the $\mathrm{Ba}(\mathrm{A})$ mode.

tor in Jülich, Germany. The europium-151 nuclear inelastic scattering measurements have been carried out at $25 \mathrm{~K}$ on $100 \mathrm{mg}$ of $\mathrm{Eu}_{8} \mathrm{Ga}_{16} \mathrm{Ge}_{30}$ on beam line ID22N (Ref. 16) at the European Synchrotron Radiation Facility in Grenoble, France. The powder filled a slit in a copper block which was covered with capton and placed upside-down on the cold finger of a cryostat. The photodiode collecting the fluorescence products of the europium-151 nuclear deexcitation was located $\approx 1 \mathrm{~cm}$ below the sample. The temperature of the sample during measurement has been determined by the detailed balance analysis of the inelastic spectrum.

\section{RESULTS AND DISCUSSION}

The experimentally weighted phonon densities of states, DOS, for $\mathrm{Ba}_{8} \mathrm{Ga}_{16} \mathrm{Ge}_{30}$ and $\mathrm{Sr}_{8} \mathrm{Ga}_{16} \mathrm{Ge}_{30}$, see Fig. 1, have been extracted from the inelastic neutron scattering data by integration of the scattering over a $2 \theta$ range of $30^{\circ}-130^{\circ}$ and with a subsequent subtraction of the background. The observation that the local mode is independent of the integration range chosen is expected for a dispersion free optical mode. In contrast to the study of filled skutterudites ${ }^{6}$ for which there is an unfilled reference compound, there is no reference compound in which the clathrate $R(\mathrm{~A})$ and $R(\mathrm{~B})$ cages are empty. As a consequence, the DOS for $\mathrm{Ba}_{8} \mathrm{Ga}_{16} \mathrm{Ge}_{30}$ and $\mathrm{Sr}_{8} \mathrm{Ga}_{16} \mathrm{Ge}_{30}$ have been fit, see Table I, between 0 and $15 \mathrm{meV}$ with a simplified three term phenomenological model given by

$$
\operatorname{DOS}(\omega)=a \omega^{2}+b \mathrm{G}\left(E_{R}, w_{R}\right)+c \mathrm{G}\left(E_{O}, w_{O}\right),
$$

where $\mathrm{G}(E, w)$ is a normalized Gaussian function. The first parabolic term corresponds to the Debye model for acoustic phonons, the second term, a Gaussian peak centered on $E_{R}$ with a full-width at half maximum of $w_{R}$, is associated with the $R(\mathrm{~B})$ Einstein oscillator and the third term, a broad Gaussian peak centered on $E_{O}$ with a full-width at half maximum of $w_{O}$, is associated with an ensemble of optical phonons that correspond to the GaGe framework vibrations, ${ }^{17}$ vibrations which make an important contribution to the phonon density of states because of the large number of atoms in the framework, i.e., 46 out of the 54 atoms in the unit cell are in the framework.

Because there are two different guests, $R(\mathrm{~A})$ and $R(\mathrm{~B})$, in the $R_{8} \mathrm{Ga}_{16} \mathrm{Ge}_{30}$ clathrates, it is not a priori obvious to which guest the peak at an $E_{R}$ of $\sim 4.5 \mathrm{meV}$ should be assigned. However, this peak can be assigned to the $R(\mathrm{~B})$ guest on the basis of two arguments. First, the $R(\mathrm{~B})$ stoichiometric contribution, and hence its expected scattering area, should be three times that of $R(\mathrm{~A})$. Second, because the $R(\mathrm{~A})$ guest is located in the smaller, dodecahedral, cage, both its Einstein energy and force constant are expected to be larger than those of the $R(\mathrm{~B})$ guest in the larger cage. Hence, if the peak at $\sim 4.5 \mathrm{meV}$ had been assigned to $R(\mathrm{~A})$, then one would have expected a larger $R(\mathrm{~B})$ peak at lower energy, a peak that is not observed. Further, the $R(\mathrm{~B})$ Einstein energies obtained with the present assignment agree well both with the energies obtained earlier from atomic displacement, ${ }^{8}$ heat capacity, ${ }^{8}$ and Raman scattering ${ }^{9}$ measurements, see Table II, and with the phonon dispersion calculated for the hypothetical compound, $\mathrm{Sr}_{6} \mathrm{Ge}_{46}$, ${ }^{17}$ in which the $\mathrm{Sr}$ was assumed to be found only at the center of the cage containing $\mathrm{Sr}(\mathrm{B})$. Apparently, the contribution of the $R(\mathrm{~A})$ guest to the DOS, as

TABLE I. The localized mode $E_{R}$ and $w_{R}$ values for the $R(\mathrm{~B})$ Einstein oscillator and the optical phonon $E_{O}$ and $w_{O}$ values.

\begin{tabular}{lcccc}
\hline \hline Compound & $E_{R}(\mathrm{meV})$ & $w_{R}(\mathrm{meV})^{\mathrm{a}}$ & $E_{O}(\mathrm{meV})$ & $w_{O}(\mathrm{meV})^{\mathrm{a}}$ \\
\hline $\mathrm{Ba}_{8} \mathrm{Ga}_{16} \mathrm{Ge}_{30}$ & $4.9 \pm 0.1$ & $1.5 \pm 0.1$ & $10.7 \pm 0.1$ & $3.0 \pm 0.1$ \\
$\mathrm{Sr}_{8} \mathrm{Ga}_{16} \mathrm{Ge}_{30}$ & $4.4 \pm 0.1$ & $2.0 \pm 0.1$ & $10.1 \pm 0.1$ & $3.4 \pm 0.1$ \\
\hline \hline
\end{tabular}

The instrumental resolution is $\sim 1.2$ and $2.6 \mathrm{meV}$ at an energy transfer of 5 and $10 \mathrm{meV}$, respectively. 
TABLE II. The Einstein energies, ${ }^{a}$ in $\mathrm{meV}$, of the guests in $R_{8} \mathrm{Ga}_{16} \mathrm{Ge}_{30}$ obtained by different experimental techniques.

\begin{tabular}{|c|c|c|c|c|c|c|c|}
\hline \multirow[b]{3}{*}{ Technique } & \multicolumn{6}{|c|}{ Compound } & \multirow[b]{3}{*}{ Ref. } \\
\hline & \multicolumn{2}{|c|}{$\mathrm{Ba}_{8} \mathrm{Ga}_{16} \mathrm{Ge}_{30}$} & \multicolumn{2}{|c|}{$\mathrm{Sr}_{8} \mathrm{Ga}_{16} \mathrm{Ge}_{30}$} & \multicolumn{2}{|c|}{$\mathrm{Eu}_{8} \mathrm{Ga}_{16} \mathrm{Ga}_{30}$} & \\
\hline & $\mathrm{Ba}(\mathrm{A})$ & $\mathrm{Ba}(\mathrm{B})$ & $\operatorname{Sr}(A)$ & $\operatorname{Sr}(\mathrm{B})$ & $\mathrm{Eu}(\mathrm{A})$ & $\mathrm{Eu}(\mathrm{B})$ & \\
\hline Heat capacity & \multicolumn{2}{|c|}{$5.2^{\mathrm{b}}$} & \multicolumn{2}{|c|}{$4.6^{\mathrm{b}}$} & \multicolumn{2}{|r|}{$2.6^{\mathrm{b}}$} & 8 \\
\hline & \multicolumn{2}{|c|}{$6.9^{\mathrm{b}}$} & \multicolumn{2}{|c|}{$6.8^{\mathrm{b}}$} & & & 4 \\
\hline & 6.7 & 3.3 & & & & & 20 \\
\hline Raman scattering & & & Inactive & $4.0,7.0,8.1^{\mathrm{c}}$ & Inactive & $2.8,6.0,6.9^{\mathrm{c}}$ & 9 \\
\hline \multirow[t]{4}{*}{ Atomic displacement } & & 5.5 & & 6.9 & & & 8 \\
\hline & & & 6.4 & 8.9 & 7.1 & 4.6 & 2 \\
\hline & 10.4 & 6.2 & & & 6.5 & 3.9 & 4 \\
\hline & 10.7 & $6.3,8.7$ & 13 & $9.0,14.1$ & & & 18 \\
\hline Resonant ultrasound & & & \multicolumn{2}{|c|}{$4.8^{\mathrm{b}}$} & \multicolumn{2}{|c|}{$1.9^{\mathrm{b}}$} & 21 \\
\hline spectroscopy & & & \multicolumn{2}{|c|}{$3.9^{\mathrm{b}}$} & & & 22 \\
\hline EXAFS & & & $8.2-8.8$ & $\begin{array}{c}10.9-11.0 \\
12.7-13.4^{\mathrm{d}}\end{array}$ & $6.7-7.1$ & $8.0-8.2$ & 23 \\
\hline Density functional theory & & & & $4.0^{\mathrm{e}}$ & & & 16 \\
\hline Inelastic neutron scattering & & 4.7 & & 4.2 & & & This work \\
\hline Nuclear inelastic scattering & & & & & 6.7 & $3.0,4.9,7.5$ & This work \\
\hline
\end{tabular}

${ }^{\mathrm{a}} \mathrm{A}$ conversion factor of $0.08617 \mathrm{meV} / \mathrm{K}$ has been used herein.

${ }^{\mathrm{b}}$ Attributed to the B site, although no separation of the contribution of the A and B sites was reported.

'Only the lowest of the modes is clearly assigned to the "rattler" and only the three lowest observed modes are listed.

"Values obtained under the assumption that $25 \%$ of the $\operatorname{Sr}(\mathrm{B})$ are "on-center."

${ }^{\text {e}}$ Calculated for the $\mathrm{Sr}_{6} \mathrm{Ge}_{46}$ hypothetical compound.

measured by neutron scattering, cannot be separated from the intense contribution of the lowest energy optical phonons that are centered at $E_{O}$. Further, because the potential for $R(\mathrm{~B})$ in the larger cage is anisotropic ${ }^{18}$ it is likely that the contribution observed at the energy $E_{R}$, corresponds only to the lowest optical mode associated with $R(\mathrm{~B})$; the contributions of the higher energy modes being hidden in the broad peak around $E_{O}$, corresponding to the framework optical phonons. ${ }^{17}$

Surprisingly, the Einstein energy, $E_{R}$, of the $\mathrm{Ba}(\mathrm{B})$ localized mode is $\sim 10 \%$ larger than that of $\operatorname{Sr}(B)$. The frequency, $\nu$, of a harmonic oscillator is proportional to $\sqrt{k} / \mathrm{m}$, where $k$ is the bonding strength and $m$ is the oscillator mass. If one assumes an equal bonding strength, a $\nu_{\mathrm{Ba}(\mathrm{B})} / \nu_{\mathrm{Sr}(\mathrm{B})}$ ratio of $\sim 0.8$ is expected, whereas a ratio of $\sim 1.1$ is actually observed. Consequently, the effective coupling constant for this mode, $k_{\mathrm{Ba}(\mathrm{B})}$, is likely to be $\sim 1.9$ times larger than that of $k_{\mathrm{Sr}(\mathrm{B})}$. No definitive conclusion can be drawn so far about the relative bonding of $\mathrm{Ba}$ and $\mathrm{Sr}$ in their clathrate cages because the observed off-center placement ${ }^{19}$ of the Sr yields highly anisotropic bonding ${ }^{18}$ with its clathrate cage framework. This larger anisotropy in the $\mathrm{Sr}$ bonding is also consistent with the observation that the $R(\mathrm{~B})$ localized mode is broader in $\mathrm{Sr}_{8} \mathrm{Ga}_{16} \mathrm{Ge}_{30}$ than in $\mathrm{Ba}_{8} \mathrm{Ga}_{16} \mathrm{Ge}_{30}$, see Table I. Our observation of stronger bonding of $\mathrm{Ba}$ than $\mathrm{Sr}$ to the clathrate framework agrees with the heat capacity ${ }^{4,8}$ results but disagrees with the observation by Bentien et al. ${ }^{18}$ However, as pointed out in Ref. 20 and illustrated in Table II, there is a discrepancy between Einstein energies derived from different physical techniques, such as atomic displacement, heat capacity, and spectroscopic techniques. In particular, the data obtained herein from inelastic neutron scattering reveals only the lowest energy optical mode for $\mathrm{Sr}(\mathrm{B})$ and $\mathrm{Ba}(\mathrm{B})$, the mode most likely associated with vibrations in the direction perpendicular to the off-centering plane. It is quite possible that this perpendicular mode is softer for the $\mathrm{Sr}(\mathrm{B})$ rattler than for the $\mathrm{Ba}(\mathrm{B})$ rattler, whereas the in-plane optical modes at higher energies, corresponding to the other components of the oscillator strength tensor, are harder for $\mathrm{Sr}(\mathrm{B})$ than for $\mathrm{Ba}(\mathrm{B})$. This might explain why the $\mathrm{Sr}(\mathrm{B})$ Einstein energies reported in Ref. 18 and 23 are larger than those observed by other techniques.

The nature of the guest-framework interactions in $\mathrm{Sr}_{8} \mathrm{Ga}_{16} \mathrm{Ge}_{30}$ and $\mathrm{Ba}_{8} \mathrm{Ga}_{16} \mathrm{Ge}_{30}$ has been theoretically described $^{24}$ as an electrostatic interaction between essentially divalent strontium or barium cations and their cage. The calculated charge density of the guest cation is larger in the A cage than in the $B$ cage and the charge densities of the barium cations are larger than those of the strontium cations in both the A and B cages. These calculations indicate that, as expected, the bonds in the smaller A cages are stronger than the bonds in the larger B cages and that the barium guests are more strongly bound to their cage framework than are the strontium guests, a conclusion that is in agreement with the larger effective coupling constant observed herein for barium. Further, the displacement of the guest cations from the center of the larger cages leads to the formation of guest-framework interactions that are even stronger and of the same order as those observed in the smaller cages. As 


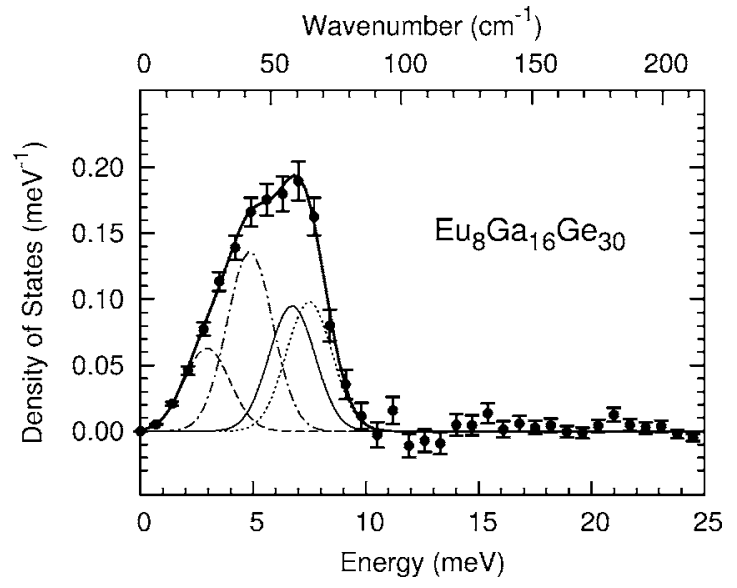

FIG. 2. The europium partial phonon density of states in $\mathrm{Eu}_{8} \mathrm{Ga}_{16} \mathrm{Ge}_{30}$ obtained by nuclear inelastic scattering. The bold solid line is a fit with Eq. (2), the solid line represents the contribution of the $\mathrm{Eu}(\mathrm{A})$ "rattling" mode, and the dashed, dotted-dashed, and dotted lines represent the contributions of the $\mathrm{Eu}(\mathrm{B}, 1), \mathrm{Eu}(\mathrm{B}, 2)$, and $\mathrm{Eu}(\mathrm{B}, 3)$ "rattling" modes, respectively.

recently pointed out by Bentien et al. ${ }^{18}$ it should be noted that earlier crystallographic studies ${ }^{25,26}$ and recent thermal conductivity measurements ${ }^{20}$ suggest that the $\mathrm{Ba}(\mathrm{B})$ guest can also be off-center in the $p$-type doped $\mathrm{Ba}_{8} \mathrm{Ga}_{16} \mathrm{Ge}_{30}$. Hence, it would be of interest to study the influence that doping has on the phonon density of states in this compound.

As indicated above, the behavior of the guests in $\mathrm{Eu}_{8} \mathrm{Ga}_{16} \mathrm{Ge}_{30}$ cannot be studied by inelastic neutron scattering because europium has a large neutron absorption cross section. Fortunately, europium- 151 is a Mössbauer active nuclide, and the europium partial phonon density of states can be obtained by nuclear inelastic scattering measurements. ${ }^{14}$ This technique also has the added advantage that the optical phonon modes that do not involve europium do not contribute to the observed scattering. Thus, in contrast to the inelastic neutron scattering measurements for the $\mathrm{Ba}_{8} \mathrm{Ga}_{16} \mathrm{Ge}_{30}$ and $\mathrm{Sr}_{8} \mathrm{Ga}_{16} \mathrm{Ge}_{30}$ clathrates reported above, the nuclear inelastic scattering measurements provide an ideal technique for completely separating the vibrational modes of the $\mathrm{Eu}(\mathrm{A})$ and $\mathrm{Eu}(\mathrm{B})$ guests from the framework vibrational modes in $\mathrm{Eu}_{8} \mathrm{Ga}_{16} \mathrm{Ge}_{30}$.

The nuclear inelastic scattering data obtained for the $\mathrm{Eu}_{8} \mathrm{Ga}_{16} \mathrm{Ge}_{30}$ clathrate have been analyzed with the INES program implemented in IDL code according to the theory of Kohn and Chumakov. ${ }^{27}$ In materials with a soft, low lying, optical mode, a significant multiphonon peak is known to lie under the elastic peak at zero energy. ${ }^{14,28}$ Hence it is important in such materials to record the nuclear inelastic scattering at low temperature, and to carefully extract the elastic peak by using the method described in Ref. 27.

The europium partial phonon density of states obtained at $25 \mathrm{~K}$ is shown in Fig. 2. The contributions of the $\mathrm{Eu}(\mathrm{A})$ and $\mathrm{Eu}(\mathrm{B})$ rattling modes are not clearly separated, even with the achieved instrumental resolution of $1.3 \mathrm{meV}$, which is state of the art monochromatization at the energy of $25 \mathrm{keV}$ and only available at third generation sources like the ESRF. The peaks in the phonon density of states are broadened at least
TABLE III. The relative area, $a$, energy, $E$, and width, $w$, for the localized modes associated with the two europium rattlers in $\mathrm{Eu}_{8} \mathrm{Ga}_{16} \mathrm{Ge}_{30}$.

\begin{tabular}{llccc}
\hline \hline Model & Guest & $a(\%)$ & $E(\mathrm{meV})$ & $w(\mathrm{meV})$ \\
\hline 2 modes & $\mathrm{Eu}(\mathrm{A})$ & $65 \pm 10$ & $6.7 \pm 0.2$ & $3.2 \pm 0.4$ \\
& $\mathrm{Eu}(\mathrm{B})$ & $35 \pm 10$ & $3.8 \pm 0.3$ & $3.0 \pm 0.2$ \\
4 modes & $\mathrm{Eu}(\mathrm{A})$ & $24 \pm 20$ & $6.7 \pm 2.5$ & $2.4 \pm 0.2^{\mathrm{a}}$ \\
& $\mathrm{Eu}(\mathrm{B}, 1)$ & $16 \pm 4$ & $3.0 \pm 0.2$ & $\mathrm{a}$ \\
& $\mathrm{Eu}(\mathrm{B}, 2)$ & $35 \pm 10$ & $4.9 \pm 0.5$ & $\mathrm{a}$ \\
& $\mathrm{Eu}(\mathrm{B}, 3)$ & $25 \pm 20$ & $7.5 \pm 1.2$ & $\mathrm{a}$ \\
\hline \hline
\end{tabular}

aThe full-width at half maximum, $w$, was constrained to be the same for all four components.

in part because of the GaGe disorder on the clathrate framework. Because of this broadening and the overlap of the europium modes, several physically reasonable models have been used to fit the data shown in Fig. 2. The first statistically satisfactory and simplest fitting model used two Gaussian peaks at 3.8 and $6.7 \mathrm{meV}$ with a full-width at half-maxium of 3.0 and $3.2 \mathrm{meV}$, respectively. These energies are in fair agreement with the energies obtained from various experimental measurements, see Table II, for the $\mathrm{Eu}(\mathrm{B})$ and $\mathrm{Eu}(\mathrm{A})$ rattler modes, respectively. However, the ratio in the areas of $\mathrm{Eu}(\mathrm{B}): \mathrm{Eu}(\mathrm{A})$ of $1: 2$ instead of the 3:1 expected from the stoichiometry and the broad linewidth of twice the instrumental resolution suggest that, as could be expected from the anisotropy of the potential in the $\mathrm{Eu}(\mathrm{B})$ cage, different vibrational modes are observed for the $\mathrm{Eu}(\mathrm{B})$ rattling modes. The resulting parameters obtained from this first fitting model are given in Table III for comparison with those obtained with the more elaborate and physically reasonable fitting model described below.

The vibrational modes of $\mathrm{Eu}(\mathrm{A})$ and $\mathrm{Eu}(\mathrm{B})$ have already been investigated by various techniques and the resulting Einstein energies are summarized in Table II. The $\mathrm{Eu}(\mathrm{A})$ vibrational mode is a Raman silent mode, and in an approximation in which the GaGe disorder on the dodecahedron is neglected, this mode is a triply degenerate $T_{1 u}$ mode, see for example Ref. 29. The anisotropy of the potential in the $\mathrm{Eu}(\mathrm{B})$ cage probably yields several vibrational modes which are Raman active. ${ }^{9}$ EXAFS measurements ${ }^{23}$ yield information both on the $\mathrm{Eu}(\mathrm{A})$ and the $\mathrm{Eu}(\mathrm{B})$ vibrational modes, however only the $\mathrm{Eu}(\mathrm{B})$ vibration with the highest frequency can be determined. Finally, because the $\mathrm{Eu}(\mathrm{B})$ guests are off-center in the large cage, three different vibrational modes, corresponding to three oscillator strength components, are expected probably at significantly different energies.

Hence, a model in which the phonon DOS is expressed as the sum of one $\mathrm{Eu}(\mathrm{A})$ and three $\mathrm{Eu}(\mathrm{B})$ Gaussians contributions has been developed,

$$
\operatorname{DOS}(\omega)=a_{\mathrm{Eu}(\mathrm{A})} \mathrm{G}\left(\omega, E_{\mathrm{Eu}(\mathrm{A})}, w\right)+\sum_{i} a_{\mathrm{Eu}(\mathrm{B}, i)} \mathrm{G}\left(\omega, E_{\mathrm{Eu}(\mathrm{B}, i)}, w\right),
$$


where $\mathrm{G}\left(\omega, E_{\mathrm{Eu}}, w\right)$ is a normalized Gaussian function centered at $E_{\mathrm{Eu}}$ with a full-width at half maximum of $w$, and $i$ $=1,2$, and 3 . In order to limit the number of fit parameters, all components have been constrained to have the same linewidth, $w$. Because the density of states is normalized, there are three different $a$ parameters for the three relative areas and four $E_{\mathrm{Eu}}$ parameters for the four vibrational energies. In view of the limited resolution of the data, a fit with seven free parameters may not be reasonable. Hence, in an initial fit, some parameters were constrained to be in agreement with previously determined ${ }^{9,23}$ values. First, the energy of the lowest $E_{\mathrm{Eu}(\mathrm{B}, 1)}$ vibrational mode was fixed at $2.8 \mathrm{meV}$, the energy ${ }^{9}$ of the lowest Raman active mode; second, the energy of the $E_{\mathrm{Eu}(\mathrm{A})}$ vibrational mode was fixed at $6.9 \mathrm{meV}$, as observed $^{23}$ by EXAFS. The $E_{\mathrm{Eu}(\mathrm{B}, 2)}$ and $E_{\mathrm{Eu}(\mathrm{B}, 3)}$ initial values were set at 4.3 and $7.8 \mathrm{meV}$, and three equal relative areas were used as initial parameters. In a second fit, these constrains were released, but the final fitted values given in Table III remained close to the initially constrained values. The four fitted Einstein energies are in good agreement with previously reported values, see Table II, and more specifically with the three lowest energy Raman lines ${ }^{9}$ and the energies obtained by EXAFS. ${ }^{23}$

Several other fits with three Gaussians or with various constrains were attempted but they all proved either significantly poorer than the fit shown in Fig. 2 or gave values in disagreement with previously reported energies for the vibrational modes. Most importantly, no vibrational mode above $\sim 8 \mathrm{meV}$ or $93 \mathrm{~K}$ with any significant contribution above five percent can be incorporated into the fitting model. This indicates that neither europium guests participate in any vibrational modes above an energy of $\sim 9 \mathrm{meV}$, i.e., the europium vibrational modes are fully decoupled from the higher energy optical modes of the GaGe framework. Whether europium guests participate significantly in the acoustic modes of the materials can only be decided by a study of the actual phonon dispersion in a single crystal, a study that is not possible by neutron scattering, nor by nuclear inelastic scattering.

As is reflected by the large error bars on the $E_{\mathrm{Eu}(\mathrm{A})}$, $E_{\mathrm{Eu}(\mathrm{B}, 3)}, a_{\mathrm{Eu}(\mathrm{A})}$, and $a_{\mathrm{Eu}(\mathrm{B}, 3)}$ parameters, see Table III, the $\mathrm{Eu}(\mathrm{A})$ and $\mathrm{Eu}(\mathrm{B}, 3)$ vibrational modes cannot be clearly separated, and the relative contributions are impossible to determine. The overall linewidth, $w$, is however determined rather accurately, because it is strongly constrained by the sharp slope in the density of states between 8 and $9 \mathrm{meV}$. Because the instrumental resolution of $1.3 \mathrm{meV}$ is significantly smaller than the observed width of $2.4 \mathrm{meV}$ it is likely that the observed peaks have some significant intrinsic broadening, as is indicated by the lack of any feature with an $\sim 1.3 \mathrm{meV}$ resolution in the observed data. It is hence not obvious that any future improvement in the resolution through an enhancement of the high resolution monochromators will yield a better peak separation. Finally, it should be noted that the $\mathrm{Eu}(\mathrm{B})$ Einstein energies are a very important parameter in determining the tunneling splitting of the multiple well potential in which the off-center europium guests reside. ${ }^{21}$

\section{CONCLUSIONS}

The presence of low-lying localized vibrational modes in the $\mathrm{Ba}_{8} \mathrm{Ga}_{16} \mathrm{Ge}_{30}, \mathrm{Sr}_{8} \mathrm{Ga}_{16} \mathrm{Ge}_{30}$, and $\mathrm{Eu}_{8} \mathrm{Ga}_{16} \mathrm{Ge}_{30}$ filled clathrates has been confirmed by the microscopic determination of their phonon densities of states through neutron inelastic scattering and nuclear inelastic scattering measurements. Similar low-energetic rattling modes have been reported previously in the filled skutterudites, ${ }^{5,6}$ another important class of thermoelectric materials. Because of the resonant nature of the nuclear inelastic scattering technique, the determination of the phonon DOS in $\mathrm{Eu}_{8} \mathrm{Ga}_{16} \mathrm{Ge}_{30}$ provides indisputable evidence that the "rattling" guests neither interact nor participate significantly in any high energy vibrational modes.

Therefore, in view of the phonon-glass electron-crystal paradigm, the crucial mechanism that leads to a reduced thermal conductivity has to be found in the interaction and hybridization of the low-energetic rattling modes with the propagating acoustic modes of the framework. Such resonant scattering mechanisms have previously been suggested to dominate in Eu bearing clathrates for $T>10 \mathrm{~K} .{ }^{30}$ Another more or less important part of the reduction in thermal conductivity in solids containing "rattlers" may be ascribed to electron-phonon scattering mechanisms, ${ }^{31}$ in particular for $T<10 \mathrm{~K}$ in Eu bearing clathrates. ${ }^{30}$ Such electron-phonon scattering should affect and reduce to some extent both thermal and electric conductivity, and therefore such a mechanism is unlikely to play a key role for thermoelectric effects. Further detailed theoretical studies are certainly desirable and may confirm such a postulated hybridization between the lower lying acoustic modes of the framework and the local "rattler" modes. Experimentally, this mechanism can be verified by an inelastic neutron scattering study of the phonons in single crystals and such experiments are currently under preparation.

Note added in proof. After acceptance of the manuscript, we became aware of Ref. 32 in which broadening of a localized vibrational mode was observed. The fact that, in the clathrates studied herein, the local modes of both $\mathrm{Ba}$ - and Sr-guest atoms exhibit a linewidth that exceeds the instrumental resolution indicates a damping of these local modes by interactions with the surrounding lattice. The observed linewidth for the $\mathrm{Sr}$ local mode is larger than for the Ba local mode, a difference which indicates a more pronounced interaction of the Sr guests with the surrounding lattice.

\section{ACKNOWLEDGMENTS}

The authors acknowledge Dr. V. Keppens for helpful discussions. We acknowledge the support of the European Community-Access to Research Infrastructure action of the Improving Human Potential Programme (HPRI-2001-00175) for provision of neutron scattering beam time on project No. DNS-02-005. The European Synchrotron Radiation Facility is acknowledged for provision of synchrotron radiation facilities at beam line ID22N. 
*On leave at the Department of Material Science and Engineering, University of Tennessee, Knoxville, Tennessee 37996-2200, USA.

${ }^{\dagger}$ Current address: Deutsches Elektronen Synchrotron, Notkestrasse 85, D-22607 Hamburg, Germany.

${ }^{1}$ J. L. Cohn, G. S. Nolas, V. Fessatidis, T. H. Metcalf, and G. A. Slack, Phys. Rev. Lett. 82, 779 (1999).

${ }^{2}$ G. S. Nolas, T. J. R. Weakley, J. L. Cohn, and R. Sharma, Phys. Rev. B 61, 3845 (2000).

${ }^{3}$ B. C. Chakoumakos, B. C. Sales, D. Mandrus, and G. S. Nolas, J. Alloys Compd. 296, 80 (2000).

${ }^{4}$ S. Paschen, W. Carrillo-Cabrera, A. Bentien, V. H. Tran, M. Baenitz, Yu. Grin, and F. Steglich, Phys. Rev. B 64, 214404 (2001).

${ }^{5}$ V. Keppens, D. Mandrus, B. C. Sales, B. C. Chakoumakos, P. Day, R. Coldea, M. B. Maple, D. A. Gajewski, E. J. Freeman, and S. Bennington, Nature (London) 395, 876 (1998).

${ }^{6}$ R. P. Hermann, R. Jin, W. Schweika, F. Grandjean, D. Mandrus, B. C. Sales, and G. J. Long, Phys. Rev. Lett. 90, 135505 (2003).

${ }^{7}$ A. Einstein, Ann. Phys. 22, 180 (1907).

${ }^{8}$ B. C. Sales, B. C. Chakoumakos, R. Jin, J. R. Thompson, and D. Mandrus, Phys. Rev. B 63, 245113 (2001).

${ }^{9}$ G. S. Nolas and C. A. Kendziora, Phys. Rev. B 62, 7157 (2000).

${ }^{10}$ G. S. Nolas, J. L. Cohn, G. A. Slack, and S. B. Schujman, Appl. Phys. Lett. 73, 178 (1998).

${ }^{11}$ M. Seto, Y. Yoda, S. Kikuta, X. W. Zhang, and M. Ando, Phys. Rev. Lett. 74, 3828 (1995).

${ }^{12}$ W. Sturhahn, T. S. Toellner, E. E. Alp, X. Zhang, M. Ando, Y. Yoda, S. Kikuta, M. Seto, C. W. Kimball, and B. Dabrowski, Phys. Rev. Lett. 74, 3832 (1995).

${ }^{13}$ A. I. Chumakov, R. Rüffer, O. Leupold, and I. Sergueev, Struct. Chem. 14, 109 (2003).

${ }^{14}$ G. J. Long, R. P. Hermann, F. Grandjean, E. E. Alp, W. Sturhahn, C. E. Johnson, D. E. Brown, O. Leupold, and R. Rüffer, Phys. Rev. B 71, 140302(R) (2005).

${ }^{15}$ A. I. Chumakov and W. Sturhahn, Hyperfine Interact. 123/124, 781 (1999).

${ }^{16} \mathrm{http} / / / \mathrm{www}$. esrf.fr/exp_facilities/ID18/

${ }^{17}$ J. Dong, O. F. Sankey, and C. W. Myles, Phys. Rev. Lett. 86,
2361 (2000).

${ }^{18}$ A. Bentien, E. Nishibori, S. Paschen, and B. B. Iversen, Phys. Rev. B 71, 144107 (2005).

${ }^{19}$ B. C. Chakoumakos, B. C. Sales, and D. Mandrus, J. Alloys Compd. 322, 127 (2001).

${ }^{20}$ A. Bentien, M. Christensen, J. D. Bryan, A. Sanchez, S. Paschen, F. Steglich, G. D. Stucky, and B. Iversen, Phys. Rev. B 69, 045107 (2004).

${ }^{21}$ I. Zerec, V. Keppens, M. A. McGuire, D. Mandrus, B. C. Sales, and P. Thalmeier, Phys. Rev. Lett. 92, 185502 (2004).

${ }^{22}$ V. Keppens, M. A. McGuire, A. Teklu, C. Laermans, B. C. Sales, D. Mandrus, and B. C. Chakoumakos, Physica B 316-317, 95 (2002).

${ }^{23}$ R. Baumbach, F. Bridges, L. Downward, D. Cao, P. Chesler, and B. C. Sales, Phys. Rev. B 71, 024202 (2005).

${ }^{24}$ C. Gatti, L. Bertini, N. P. Blake, and B. B. Iversen, Chem.-Eur. J. 9, 4556 (2003).

${ }^{25}$ A. Bentien, A. E. C. Palmqvist, J. D. Bryan, S. Latturner, G. D. Stucky, L. Furenlid, and B. B. Iversen, Angew. Chem., Int. Ed. 39, 3613 (2000).

${ }^{26}$ A. Bentien, B. B. Iversen, J. D. Bryan, G. D. Stucky, A. E. C. Palmqvist, A. J. Schultz, and R. W. Henning, J. Appl. Phys. 91, 5694 (2002).

${ }^{27}$ V. G. Kohn and A. I. Chumakov, Hyperfine Interact. 125, 205 (2000).

${ }^{28}$ A. Barla, R. Rüffer, A. I. Chumakov, J. Metge, J. Plessel, and M. M. Abd-Elmeguid, Phys. Rev. B 61, R14881 (2000).

${ }^{29}$ E. Kroumova, M. I. Aroyo, J. M. Perez Mato, A. Kirov, C. Capillas, S. Ivantchev, and H. Wondratschek, Phase Transitions 76, 155 (2003); in particular http://www.cryst.ehu.es/rep/sam.html

${ }^{30}$ A. Bentien, V. Pacheco, S. Paschen, Yu. Grin, and S. Steglich, Phys. Rev. B 71, 165206 (2005).

${ }^{31}$ B. C. Sales, B. C. Chakoumakos, and D. Mandrus, Phys. Rev. B 61, 2475 (2000).

${ }^{32}$ J. Baumert, C. Gutt, V. P. Shpakov, J. S. Tse, M. Krisch, M. Müller, H. Requardt, D. D. Klug, S. Janssen, and W. Press, Phys. Rev. B 68, 174301 (2003). 